International Journal of Applied Mathematics

Volume 34 No. 1 2021, 111-125

ISSN: $1311-1728$ (printed version); ISSN: 1314-8060 (on-line version)

doi: http://dx.doi.org/10.12732/ijam.v34i1.5

\title{
NUMERICAL SOLUTION OF THE TIME-FRACTIONAL DIFFUSION EQUATIONS VIA QUARTER-SWEEP PRECONDITIONED GAUSS-SEIDEL METHOD
}

\author{
Andang Sunarto ${ }^{1}$, Jumat Sulaiman ${ }^{2}$ \\ Jackel Chew Vui Lung $3 \S$ \\ ${ }^{1}$ IAIN Bengkulu, Indonesia \\ Jl Raden Fatah Kota Bengkulu \\ Bengkulu, INDONESIA \\ ${ }^{2}$ Faculty of Science and Natural Resource \\ Universiti Malaysia Sabah \\ 88400, Kota Kinabalu, Sabah, MALAYSIA \\ ${ }^{3}$ Faculty of Computing and Informatics \\ Universiti Malaysia Sabah Labuan International Campus \\ 87000, Labuan F.T., MALAYSIA
}

\begin{abstract}
In this research, we propose the approximate solution of the timefractional diffusion equation based on a quarter-sweep implicit finite difference approximation equation. To derive this approximation equation, Caputo's time-fractional derivative has been used to discretize the proposed problems. By using the Caputo finite difference approximation equation, a linear system will be generated and solved iteratively. In addition to that, formulation and implementation the QSPGS iterative method are also presented. Based on the numerical results of the proposed iterative method, it can be concluded that the proposed iterative method is superior to the FSPGS and HSPGS iterative method.
\end{abstract}

AMS Subject Classification: 26A33, 65M06, 65M22

Key Words: Caputo fractional derivative; implicit finite-difference; QSPGS method

Received: August 26, 2020

(C) 2021 Academic Publications

${ }^{\S}$ Correspondence author 


\section{Introduction}

In recent years, many studies use fractional partial differential equations (FPDEs) $[14,7,15,3,2]$ for solving fractional problems to derive numerical and/or analytical solutions. Based on iterative methods for solving a one-dimensional diffusion model with constant coefficients and analytical solutions and for an instant a fractional derivative replaces the first-order space partial derivative in a diffusion model and lead to slower diffusion [14]. Therefore, there are numerical methods proposed to solve the time-fractional diffusion equations (TFDE), such as transform methods [22], finite elements together with the method of lines [15], explicit and implicit finite difference methods [15, 24]. Nevertheless, the explicit methods are conditionally stable, these finite difference schemes are available in the literature [24].

To solve the time-fractional diffusion equations (TFDE) problem needs to be discretized. Based on the implicit finite difference scheme and Caputo fractional operator, the approximation equations can be used to construct a linear system at each time level. To solve linear systems, many researchers also have discussed the concept of iterative methods, see $[20,10,19]$ and reference therein. Besides these iterative methods, the concept of block iteration has also been introduced by [8]. Furthermore, Ibrahim and Abdullah [11], and Yousif and Evans [21] have pointed out the efficiency of block iterative methods.

For solving the large linear system, Abdullah [1] initiated Half-Sweep iteration, which is one of the most known and widely used iterative techniques to solve in solving any linear systems. Differently from the Half-sweep iteration approach, Othman and Abdullah [16] have expanded this approach to initiate the Modified Explicit Group (MEG) method based on the quarter-sweep approach. It is proved that this method is one of the most efficient block iterative methods in solving any linear system as compared with ED and EDG iterative methods. Also, another researcher has shown the capability of the quarter-sweep iteration in solving nonlinear system, see [5]. Among the existing iterative methods, the preconditioned iterative methods $[4,9]$ have been widely accepted to be one of the efficient methods for solving linear systems.

Because of the advantages of these iterative methods, this paper aims to construct and investigate the effectiveness of the Quarter-Sweep Preconditioned Gauss-Seidel (QSPGS) iterative method for solving time fractional parabolic partial differential equations (TPPDE's) based on the Caputo implicit finite difference approximation equation. In this paper, we investigate the performance of the Quarter-Sweep Preconditioned Gauss-Seidel (QSGS) iterative method for solving time-fractional parabolic partial differential equations (TPPDE's) 
based on the Caputo implicit finite difference approximation equation. To demonstrate the capability of the Quarter-Sweep Preconditioned Gauss-Seidel (QSPGS) method, we also implement the Full-Sweep Preconditioned GaussSeidel or FSPGS and Half-Sweep Preconditioned Gauss-Seidel or HSPGS iterative methods being used as a control method.

\section{Preliminaries}

To begin the derivation of the QSPGS iterative method, let us consider the time-fractional diffusion equation (TFDE's) defined as

$$
\frac{\partial^{\alpha} u(x, t)}{\partial^{\alpha} t}=a(x) \frac{\partial^{2} u(x, t)}{\partial x^{2}}+b(x) \frac{\partial u(x, t)}{\partial x}+c(x) u(x, t),
$$

where $a(x), b(x)$ and $c(x)$ are known functions or constants whereas $\alpha$ is a parameter which refers to the fractional-order of time derivative. Before to set the discretizing problem (1), let us remind some definitions from the theory of fractional calculus.

Definition 1. ([17]) The Riemann-Liouville integral operator $J^{\alpha}$ of fractional order $\alpha$ is defined as

$$
J^{\alpha} f(x)=\frac{1}{\Gamma(\alpha)} \int_{0}^{x}(x-t)^{\alpha} f(t) d t, \quad \alpha>0, x>0 .
$$

Definition 2. ([17]) The Caputo fractional derivative operator $D^{\alpha}$ of order $\alpha>0$ is defined as

$$
D^{\alpha} f(x)=\frac{1}{\Gamma(m-\alpha)} \int_{0}^{x} \frac{f^{(m)}(t)}{(x-t)^{\alpha-m+1}} d t
$$

with $m-1<\alpha \leq m, m \in \mathbb{N}, x>0$. In (2) and (3), $\Gamma(\alpha)$ is the well-known Gamma function

$$
\Gamma(\alpha)=\int_{0}^{\infty} x^{t-1} e^{-x} d x .
$$

For solving the numerical of time-fractional diffusion equation (TFDE's), in equation (1), we get numerical approximations by using the Caputo derivative definition with Dirichlet boundary conditions and consider the non-local fractional derivative operator. This approximation equation can be categorized as an unconditionally stable scheme. On strength of Problem (1), the solution 
domain of the problem has been restricted to the finite space domain $0 \leq x \leq \gamma$, with $0<\alpha<1$ whereas the parameter $\alpha$ refers to the fractional-order of space derivative. To solve Problem (1), let us consider the initial and boundary conditions of Problem (1) be given as

$$
u(0, t)=g_{0}(t), u(l, t)=g_{1}(t),
$$

and the initial condition

$$
u(x, 0)=f(x),
$$

where $g_{0}(t), g_{1}(t)$, and $f(x)$ are given functions. Based on a discretized approximation to the time-fractional derivative in equation (1), we consider Caputo's fractional partial derivative of order $\alpha$, as

$$
\frac{\partial^{\alpha} u(x, t)}{\partial^{\alpha} t}=\frac{1}{\Gamma(n-1)} \int_{0}^{\infty} \frac{\partial u(x-s)}{\partial t}(t-s)^{-\alpha} d s, t>0,0<\alpha<1 .
$$

\section{The Caputo implicit finite difference approximation}

Based on equation (7), the formulation of the Caputo fractional partial derivative of the first order approximation method is given as

$$
D_{t}^{\alpha} U_{i, n} \cong \sigma_{\alpha, k} \Sigma_{j=1}^{n} \omega_{j}^{\alpha}\left(U_{i, n-j+1}-U_{i, n-j}\right)
$$

and we have the following expressions

$$
\sigma_{\alpha, k}=\frac{1}{\Gamma(1-\alpha)(1-\alpha)^{1-\alpha}}
$$

and

$$
\omega_{j}^{\alpha}=j^{1-\alpha}-(j-1)^{1-\alpha} .
$$

First, to discretize Problem (1), let the solution domain of the problem be partitioned uniformly. To do this, we consider some positive integers $m$ and $n$ in which the grid sizes in space and time directions for the finite-difference algorithm are defined as $h=\delta x=\gamma / m$ and $k=\delta t=T / n$, respectively. According to these grid sizes, we develop the uniform grid network of the solution domain where the grid points in the space interval $[0, \gamma]$ are shown as the numbers $x_{i}=i h, i=0,1,2, \ldots, m$, and the grid points in the time interval $[0, T]$ are labeled $t_{j}=j k, j=0,1,2, \ldots, n$. Then the values of the function $U(x, t)$ at the grid points are denoted as $U_{i, j}=U\left(x_{i}, t_{j}\right)$. According to equation (8) and the implicit finite difference discretization scheme, the Caputo implicit finite 
difference approximation equation of Problem (1) to the grid point centered at $\left(x_{i}, t_{j}\right)=(i h, j k)$ is given as

$$
\begin{aligned}
\sigma_{\alpha, k} \Sigma_{j=1}^{n} \omega_{j}^{\alpha}\left(U_{i, n-j+1}-U_{i, n-j}\right) & =a_{i} \frac{1}{16 h^{2}}\left(U_{i-4, n}-2 U_{i, n}\right. \\
& \left.+U_{i+4, n}\right)+b_{i} \frac{1}{8 h}\left(U_{i+4, n}-U_{i-4, n}\right)+c_{i} U_{i, n}
\end{aligned}
$$

for $i=4,8, \ldots, m-4$. Thus, based on equation (11), this approximation equation is known as the fully implicit finite difference approximation equation which is consistent first-order accuracy in time and second-order in space. Particularly, the approximation equation (11) can be rewritten based on the specified time level. Immediately, we have for $n \geq 2$ :

$$
\sigma_{\alpha, k} \Sigma_{j=1}^{n} \omega_{j}^{\alpha}\left(U_{i, n-j+1}-U_{i, n-j}\right)=p_{i} U_{i-4, n}+q_{i} U_{i, n}+r_{i} U_{i+4, n},
$$

where

$$
p_{i}=\frac{a_{i}}{16 h^{2}}-\frac{b_{i}}{8 h}, q_{i}=c_{i}-\frac{a_{i}}{8 h^{2}}, r_{i}=\frac{a_{i}}{16 h^{2}}+\frac{b_{i}}{8 h} .
$$

Also, we get for $n=1$,

$$
-p_{i} U_{i-4,1}+q *_{i} U_{i, 1}-r_{i} U_{i+4,1}=f_{i, 1}, i=4,8, \ldots, m-4,
$$

where $\omega_{j}^{(\alpha)}=1, q *_{i}=\sigma_{\alpha, k}-q_{i}, f_{i, 1}=\sigma_{\alpha, k} U_{i, 1}$ Furthermore, based on equation (14), it can be seen that the tridiagonal linear system can be constructed in matrix form as

$$
A U=f
$$

where

$$
\begin{aligned}
& A=\left[\begin{array}{cccccc}
q *_{4} & -r_{4} & & & & \\
-P_{8} & q *_{8} & -r_{8} & & & \\
& -p_{12} & q *_{12} & -r 12 & & \\
& & \ddots & \ddots & \ddots & \\
& & & -p_{m-8} & q *_{m-8} & -r_{m-8} \\
& & & & -p_{m-4} & q *_{m-4}
\end{array}\right]_{\left(\frac{m}{4}-1\right) \times\left(\frac{m}{4}-1\right)} \\
& U=\left[\begin{array}{llllll}
U_{4,1} & U_{8,1} & U_{12,1} & \ldots & U_{m-8,1} & U_{m-4,1}
\end{array}\right], \\
& f=\left[\begin{array}{llllll}
U_{4,1}+p_{1} U_{0,1} & U_{8,1} & U_{12,1} & \ldots & U_{m-8,1} & U_{m-4,1}+p_{m-4} U_{m, 1}
\end{array}\right] .
\end{aligned}
$$




\section{Analysis of stability}

In this section, we have considered the stability analysis of the implicit finite difference approximation equation in equation (11). For stability analysis, we will use Von-Neumann's [13] and the Lax equivalence theorem [18]. It follows that the numerical solution of the approximation equation in equation (11) converges to the exact solution as $h, k \rightarrow 0$.

Theorem 3. The fully implicit numerical method (11), the solution to equation (1) with $0<\alpha<1$ on the finite domain $0 \leq x \leq 1$, with zero boundary condition $u(0, t)=u(l, t)=0$ for all $t \geq 0$ is consistent and unconditionally stable.

Proof. To examine the stability of the proposed method, we find for the solution of the form $U_{j}^{n}=\zeta_{n} e^{i \omega j h}, i=\sqrt{-1}, \omega$ is real. Therefore, equation (12) becomes

$$
\begin{aligned}
& \sigma_{\alpha, k} \zeta_{n-1} e^{i \omega j h}-\sigma_{\alpha, k} \sum_{j=2}^{n} \omega_{j}^{(\alpha)}\left(\zeta_{n-j+1} e^{i \omega j h}-\zeta_{n-j} e^{i \omega j h}\right) \\
& =-p_{i} \zeta_{n} e^{i \omega(j-4) h}+\left(\sigma_{\alpha, k}-q_{i}\right) \zeta_{n} e^{i \omega j h}-r_{i} \zeta_{n} e^{i \omega(j+4) h} .
\end{aligned}
$$

By simplifying and reordering over equation (19), we have

$$
\begin{aligned}
\sigma_{\alpha, k} \zeta_{n-1}-\sigma_{\alpha, k} \sum_{j=2}^{n} \omega_{j}^{(\alpha)}\left(\zeta_{n-j+1}-\zeta_{n-j}\right) & \\
& =\zeta_{n}\left(\left(\left(-p_{i}-r_{i}\right) \cos (\omega h)\right)+\left(\sigma_{\alpha, k}-q_{i}\right)\right) .
\end{aligned}
$$

This can be reduced to

$$
\zeta_{n}=\frac{\zeta_{n-1}+\sum_{j=2}^{n} \omega_{j}^{(\alpha)}\left(\zeta_{n-j}-\zeta_{n-j+1}\right)}{1+\frac{\left(-p_{i}-r_{i}\right)}{\sigma_{\alpha, k}} \cos (\omega h)-\frac{q_{i}}{\sigma_{\alpha, k}}} .
$$

From equation (21), it can be observed that the conducted as

$$
\left(1+\frac{\left(-p_{i}-r_{i}\right)}{\sigma_{\alpha, k}} \cos (\omega h)-\frac{q_{i}}{\sigma_{\alpha, k}}\right) \geq 1,
$$

for all $\alpha, n, \omega, h$ and $k$, we have

$$
\zeta_{1} \leq \zeta_{0}
$$

and

$$
\zeta_{n} \leq \zeta_{n-1}+\Sigma_{j=2}^{n} \omega_{j}^{(\alpha)}\left(\zeta_{n-j}-\zeta_{n-j+1}\right), \quad n \geq 2 .
$$


Thus, for $n=2$, the last inequality implies

$$
\zeta_{2} \leq \zeta_{1}+\omega_{2}^{(\alpha)}\left(\zeta_{0}-\zeta_{1}\right)
$$

Again, repeating the above process, we can get

$$
\zeta_{j} \leq \zeta_{j-1}, \quad j=1,2, \ldots, n-1 .
$$

From equation (24), we finally have

$$
\zeta_{n} \leq \zeta_{n-1}+\Sigma_{j=2}^{n} \omega_{j}^{(\alpha)}\left(\zeta_{n-j}-\zeta_{n-j+1}\right) \leq \zeta_{n-j} .
$$

Since each term in the summation is negative, it shows that the inequalities (23) and (24) imply

$$
\zeta_{n} \leq \zeta_{n-1} \leq \zeta_{n-2} \leq \ldots \leq \zeta_{1} \leq \zeta_{0}
$$

Thus

$$
\zeta_{n}=\left|U_{j}^{n}\right| \leq \zeta_{0}=\left|U_{j}^{0}\right|=\left|f_{j}\right|,
$$

which entails $\left\|U_{j}^{n}\right\| \leq\left\|f_{j}\right\|$, and we have stability.

\section{Formulation of Quarter-Sweep Preconditioned Gauss-Seidel (QSPGS)}

Concerning the tridiagonal linear system (16), the characteristics of its coefficient matrix are large scale and sparse. As mentioned in Section 1, many researchers have discussed various iterative methods, such as Gunawardena [9] and Young [20]. To obtain numerical solutions of the tridiagonal linear system (16), we consider the Quarter-Sweep Preconditioned Gauss-Seidel (QSPGS) iterative method, which is the most known and widely used for solving any linear system. Before applying the QSPGS iterative method, we need to transform the original linear system (15) into the preconditioned linear system

$$
A^{*} x=f^{*},
$$

where $A^{*}=P A P^{T}, f^{*}=P f, U=P^{T} x$. The matrix $P$ is called a preconditioned matrix and defined as [12]

$$
P=I+S,
$$


where

$$
S=\left[\begin{array}{cccccc}
0 & -r_{1} & 0 & 0 & 0 & 0 \\
0 & 0 & -r_{2} & 0 & 0 & 0 \\
0 & 0 & 0 & -r_{3} & 0 & 0 \\
0 & 0 & 0 & 0 & \ddots & 0 \\
0 & 0 & 0 & 0 & 0 & -r_{m-1} \\
0 & 0 & 0 & 0 & 0 & 0
\end{array}\right]_{(m-1) \times(m-1)}
$$

and the matrix $I$ is an identical matrix. To formulate the QSPGS method, let the coefficient matrix $A^{*}$ in $(30)$ be expressed as the summation of the three matrices

$$
A^{*}=D-L-V \text {, }
$$

where $D, L$, and $V$ are diagonal, lower triangular, and upper triangular matrices, respectively. By using equation (30), the formulation of QSPGS iterative method can be defined generally as

$$
x^{(k+1)}=(D-L)^{-1} V x^{(k)}(D-L)^{-1} f^{*},
$$

where $x^{(k+1)}$ represents an unknown vector at $(k+1)$ th iterations. The implementation of the QSPGS iterative method can be described in Algorithm 1.

\section{Algorithm 1: QSPGS}

i. Initialize $U \leftarrow 0$ and $\epsilon \leftarrow 10^{-10}$,

ii. For $j=1,2, \ldots, n$, implement

For $i=1,2, \ldots, m-1$, calculate $x^{(k+1)}=(D-L)^{-1} V x^{(k)}+(D-L)^{-1} f *$

Convergence test. If the convergence criterion i.e. $\left\|U^{(k+1)}-U^{(k)}\right\| \leq \epsilon$ is satisfied, go to Step (iii). Otherwise, go back to Step (i).

iii. Display approximate solutions.

\section{Numerical experiment}

In this section, we use one example of the time-fractional diffusion equation to show the accuracy and effectiveness properties of the Quarter Sweep Preconditioned Gauss-Seidel (QSPGS) compare Full-Sweep Preconditioned GaussSeidel (FSPGS) and Half Sweep Preconditioned Gauss-Seidel (HSPGS) iterative methods. These three parameters were executed on the computer using a program written in $C$ language. For comparison purpose, three parameters will 
be considered such as the number of iterations, execution time (in seconds), and maximum absolute error at three different values of $\alpha=0.25,0.50$, and 0.75. For the implementation of these three iterative schemes, the convergence test considered the tolerance error, which is fixed as $\epsilon=10^{-10}$. To illustrate the performance of QSPGS iteration method, let us consider the time-fractional initial boundary value problem be given as follows.

Example 1: ([6])

$$
\frac{\partial^{\alpha} u(x, t)}{\partial t^{\alpha}}=\frac{\partial^{2} u(x, t)}{\partial x^{2}}, \quad 0<\alpha \leq 1,0 \leq x \leq \gamma, t>0
$$

where the boundary conditions are stated in fractional terms

$$
u(0, t)=\frac{2 k t^{\alpha}}{\Gamma(\alpha+1)}, \quad u(l, t)=l^{2}+\frac{2 k t^{\alpha}}{\Gamma(\alpha+1)},
$$

and the initial condition $u(x, 0)=x^{2}$. Following Problem (35), as taking $\alpha=1$, it can be seen that equation (35) can be reduced to the standard diffusion equation

$$
\frac{\partial u(x, t)}{\partial t}=\frac{\partial^{2} u(x, t)}{\partial x^{2}}, \quad 0 \leq x \leq \gamma, t>0,
$$

subject to the initial condition $u(x, 0)=x^{2}$, and the boundary conditions $u(0, t)=2 k t, u(l, t)=l^{2}+2 k t$ Then, the analytical solution of equation (37) is obtained as follows:

$$
u(x, t)=x^{2}+2 k t
$$

Now, by applying the series

$$
u(x, t)=\Sigma_{n=0}^{m-1} \frac{\partial^{n} u(x, 0)}{\partial t^{n}} \frac{t^{n}}{n !}+\Sigma_{n=1}^{\infty} \Sigma_{n=0}^{m-1} \frac{\partial^{m n+i} u(x, 0)}{\partial t^{m n+i}} \frac{t^{n \alpha+i}}{\Gamma(n \alpha+i+1)}
$$

$u(x, t)$ for $0<\alpha \leq 1$, it can be shown that the analytical solution of equation (35) is given as

$$
u(x, t)=x^{2}+2 k \frac{t^{\alpha}}{\Gamma(\alpha+1)} .
$$

Example 2: ([6]) Let us consider the following time-fractional initial boundary value problem be defined as

$$
\frac{\partial^{\alpha} u(x, t)}{\partial t^{\alpha}}=\frac{1}{2} x^{2} \frac{\partial^{2} u(x, t)}{\partial x^{2}}, \quad 0<\alpha \leq 1,0 \leq x \leq \gamma, t>0,
$$


where the boundary conditions are given as $u(0, t)=0, u(1, t)=e^{t}$ and the initial condition $u(x, 0)=x^{2}$. From equation (41), as taking $\alpha=1$, it can be shown that equation (41) can also be reduced to the standard diffusion equation

$$
\frac{\partial u(x, t)}{\partial t}=\frac{1}{2} x^{2} \frac{\partial^{2} u(x, t)}{\partial x^{2}}, \quad 0 \leq x \leq \gamma, t>0 .
$$

Then, the analytical solution of equation (42) is obtained as follows:

$$
u(x, t)=x^{2} e^{t} .
$$

Now, by applying the series

$$
u(x, t)=\Sigma_{n=0}^{m-1} \frac{\partial^{n} u(x, 0)}{\partial t^{n}} \frac{t^{n}}{n !}+\Sigma_{n=1}^{\infty} \Sigma_{n=0}^{m-1} \frac{\partial^{m n+i} u(x, 0)}{\partial t^{m n+i}} \frac{t^{n \alpha+i}}{\Gamma(n \alpha+i+1)},
$$

$u(x, t)$ for $0<\alpha \leq 1$, it can be shown that the analytical solution of equation (41) is stated as

$$
u(x, t)=x^{2}\left[1+\frac{t^{\alpha}}{\Gamma(\alpha+1)}+\frac{t^{2 \alpha}}{\Gamma(2 \alpha+1)}+\frac{t^{3 \alpha}}{\Gamma(3 \alpha+1)}+\ldots\right] .
$$

All results of numerical experiments for equation (35) and (41), which were obtained from the implementation of FSPGS, HSPGS and QSPGS iterative methods have been recorded in Table 1 until Table 6 at different values of mesh sizes, $m=128,256,512,1024$, and 2048 .

\section{Conclusion}

As a conclusion for the numerical solution of the time-fractional diffusion problems, this paper deals with the implementation of the QSPGS iterative method to solve a linear system generated by the Quarter-Sweep Caputo implicit approximation equations. Through numerical experiments results from such Tables 1, 2 and 3 by comparing the performance between the FSPGS, HSPGS and QSPGS iterative methods at three different values of $\alpha=0.25,0.50$ and 0.75 , it can be seen that the percentage reduction of the number of iterations for the QSGS iterative method has declined approximately by $71.99-92.72 \%$, $50.71-95.53 \%$, and $40.41-96.45 \%$ respectively as compared with the FSPGS and HSPGS method. Implementations of computational time for the QSPGS method are much faster about $69.78-98.09 \%, 30.64-88.35 \%$, and $25.03-$ $89.99 \%$, respectively, than the FSPGS and HSPGS method. It can be concluded that the QSPGS method involves less number of iterations and computational time as compared with FSPGS and HSPGS methods. According to the accuracy of FSPGS, HSPGS, and QSPGS iterative methods, it can be stated that the numerical solutions of both methods are in good agreement. 
Table 1: Numerical result of Example 1 at $\alpha=0.25$

\begin{tabular}{ccccc}
\hline $\mathrm{M}$ & Method & $\mathrm{k}$ & time & Max Error \\
\hline \multirow{2}{*}{128} & FSPGS & 7292 & 35.86 & $9.96 \mathrm{e}-05$ \\
& HSPGS & 1966 & 5.64 & $9.96 \mathrm{e}-05$ \\
& QSPGS & 528 & 1.41 & $9.96 \mathrm{e}-05$ \\
\hline 256 & FSPGS & 26884 & 261.56 & $9.98 \mathrm{e}-05$ \\
& HSPGS & 7292 & 37.36 & $9.96 \mathrm{e}-05$ \\
& QSPGS & 1966 & 5.45 & $9.96 \mathrm{e}-05$ \\
\hline \multirow{2}{5}{12} & FSPGS & 98422 & 1916.28 & $1.00 \mathrm{e}-04$ \\
& HSPGS & 26884 & 272.45 & $9.98 \mathrm{e}-05$ \\
& QSPGS & 7292 & 35.88 & $9.96 \mathrm{e}-05$ \\
\hline 1024 & FSPGS & 357258 & 14064.44 & $1.04 \mathrm{e}-04$ \\
& HSPGS & 98422 & 2025.13 & $1.04 \mathrm{e}-04$ \\
& QSPGS & 26884 & 267.44 & $9.98 \mathrm{e}-05$ \\
\hline 2048 & FSPGS & 1183293 & 4104.17 & $1.36 \mathrm{e}-04$ \\
& HSPGS & 339197 & 3121.13 & $1.36 \mathrm{e}-04$ \\
& QSPGS & 94141 & 1120.08 & $9.98 \mathrm{e}-05$ \\
\hline
\end{tabular}

Table 2: Numerical result of Example 1 at $\alpha=0.50$

\begin{tabular}{ccccc}
\hline $\mathrm{M}$ & Method & $\mathrm{k}$ & time & Max Error \\
\hline \multirow{2}{*}{128} & FSPGS & 4715 & 2.23 & $9.84 \mathrm{e}-05$ \\
& HSPGS & 1270 & 1.59 & $9.84 \mathrm{e}-05$ \\
& QSPGS & 342 & 0.98 & $9.84 \mathrm{e}-05$ \\
\hline 256 & FSPGS & 17417 & 16.68 & $9.87 \mathrm{e}-05$ \\
& HSPGS & 4715 & 10.28 & $9.87 \mathrm{e}-05$ \\
& QSPGS & 1270 & 3.78 & $9.87 \mathrm{e}-05$ \\
\hline \multirow{2}{5}{12} & FSPGS & 63298 & 123.01 & $9.96 \mathrm{e}-05$ \\
& HSPGS & 17417 & 95.09 & $9.87 \mathrm{e}-05$ \\
& QSPGS & 4715 & 23.31 & $9.87 \mathrm{e}-05$ \\
\hline \multirow{2}{*}{024} & FSPGS & 232784 & 1007.47 & $1.03 \mathrm{e}-05$ \\
& HSPGS & 63928 & 893.24 & $9.95 \mathrm{e}-05$ \\
& QSPGS & 17417 & 171.84 & $9.87 \mathrm{e}-05$ \\
\hline 2048 & FSPGS & 1150153 & 3239.84 & $1.34 \mathrm{e}-05$ \\
& HSPGS & 232784 & 2511.66 & $1.34 \mathrm{e}-05$ \\
& QSPGS & 61246 & 975.43 & $9.98 \mathrm{e}-05$ \\
\hline
\end{tabular}


Table 3: Numerical result of Example 1 at $\alpha=0.75$

\begin{tabular}{ccccc}
\hline $\mathrm{M}$ & Method & $\mathrm{k}$ & time & Max Error \\
\hline 128 & FSPGS & 2319 & 1.93 & $1.30 \mathrm{e}-04$ \\
& HSPGS & 625 & 1.03 & $1.30 \mathrm{e}-04$ \\
& QSPGS & 170 & 0.87 & $1.30 \mathrm{e}-04$ \\
\hline 256 & FSPGS & 8585 & 12.37 & $1.30 \mathrm{e}-04$ \\
& HSPGS & 2319 & 8.23 & $1.30 \mathrm{e}-04$ \\
& QSPGS & 625 & 2.27 & $1.30 \mathrm{e}-04$ \\
\hline \multirow{2}{5}{12} & FSPGS & 31619 & 62.78 & $1.31 \mathrm{e}-04$ \\
& HSPGS & 8585 & 46.08 & $1.31 \mathrm{e}-04$ \\
& QSPGS & 2319 & 11.80 & $1.31 \mathrm{e}-04$ \\
\hline 1024 & FSPGS & 115617 & 820.93 & $1.35 \mathrm{e}-04$ \\
& HSPGS & 31619 & 636.78 & $1.35 \mathrm{e}-04$ \\
& QSPGS & 8585 & 84.30 & $1.35 \mathrm{e}-04$ \\
\hline 2048 & FSPGS & 362784 & 1305.50 & $1.35 \mathrm{e}-04$ \\
& HSPGS & 115627 & 807.13 & $1.35 \mathrm{e}-04$ \\
& QSPGS & 31691 & 629.00 & $1.35 \mathrm{e}-04$ \\
\hline
\end{tabular}

Table 4: Numerical result of Example 2 at $\alpha=0.25$

\begin{tabular}{ccccc}
\hline $\mathrm{M}$ & Method & $\mathrm{k}$ & time & Max Error \\
\hline \multirow{2}{*}{128} & FSPGS & 2873 & 8.48 & $1.95 \mathrm{e}-02$ \\
& HSPGS & 774 & 5.05 & $1.95 \mathrm{e}-02$ \\
& QSPGS & 209 & 3.42 & $1.95 \mathrm{e}-02$ \\
\hline 256 & FSPGS & 10624 & 96.54 & $1.95 \mathrm{e}-02$ \\
& HSPGS & 2873 & 19.09 & $1.95 \mathrm{e}-02$ \\
& QSPGS & 774 & 6.93 & $1.95 \mathrm{e}-02$ \\
\hline \multirow{2}{5}{12} & FSPGS & 39608 & 648.25 & $1.95 \mathrm{e}-02$ \\
& HSPGS & 10624 & 108.69 & $1.95 \mathrm{e}-02$ \\
& QSPGS & 2873 & 18.58 & $1.95 \mathrm{e}-02$ \\
\hline 1024 & FSPGS & 142635 & 791.55 & $1.95 \mathrm{e}-02$ \\
& HSPGS & 39068 & 582.43 & $1.95 \mathrm{e}-02$ \\
& QSPGS & 10624 & 107.34 & $1.95 \mathrm{e}-02$ \\
\hline 2048 & FSPGS & 487355 & 2543.23 & $1.95 \mathrm{e}-02$ \\
& HSPGS & 128676 & 1326.21 & $1.95 \mathrm{e}-02$ \\
& QSPGS & 36470 & 275.38 & $1.95 \mathrm{e}-02$ \\
\hline
\end{tabular}


Table 5: Numerical result of Example 2 at $\alpha=0.50$

\begin{tabular}{ccccc}
\hline $\mathrm{M}$ & Method & $\mathrm{k}$ & time & Max Error \\
\hline \multirow{2}{*}{28} & FSPGS & 1398 & 7.00 & $8.28 \mathrm{e}-02$ \\
& HSPGS & 378 & 5.11 & $8.28 \mathrm{e}-02$ \\
& QSPGS & 104 & 3.29 & $8.28 \mathrm{e}-02$ \\
\hline 256 & FSPGS & 5162 & 35.69 & $8.29 \mathrm{e}-02$ \\
& HSPGS & 1398 & 12.12 & $8.29 \mathrm{e}-02$ \\
& QSPGS & 378 & 6.06 & $8.28 \mathrm{e}-02$ \\
\hline \multirow{2}{*}{12} & FSPGS & 18957 & 277.23 & $8.29 \mathrm{e}-02$ \\
& HSPGS & 5162 & 57.19 & $8.29 \mathrm{e}-02$ \\
& QSPGS & 1398 & 11.90 & $8.29 \mathrm{e}-02$ \\
\hline 1024 & FSPGS & 69108 & 492.97 & $8.29 \mathrm{e}-02$ \\
& HSPGS & 38957 & 390.80 & $8.30 \mathrm{e}-02$ \\
& QSPGS & 5162 & 56.40 & $8.30 \mathrm{e}-02$ \\
\hline 2048 & FSPGS & 240051 & 1781.32 & $8.29 \mathrm{e}-02$ \\
& HSPGS & 67817 & 920.14 & $8.30 \mathrm{e}-02$ \\
& QSPGS & 19430 & 252.12 & $8.28 \mathrm{e}-02$ \\
\hline
\end{tabular}

Table 6: Numerical result of Example 2 at $\alpha=0.75$

\begin{tabular}{ccccc}
\hline $\mathrm{M}$ & Method & $\mathrm{k}$ & time & Max Error \\
\hline 128 & FSPGS & 655 & 4.44 & $1.37 \mathrm{e}-01$ \\
& HSPGS & 178 & 2.59 & $1.37 \mathrm{e}-01$ \\
& QSPGS & 50 & 1.20 & $1.37 \mathrm{e}-01$ \\
\hline 256 & FSPGS & 2420 & 15.95 & $1.37 \mathrm{e}-01$ \\
& HSPGS & 655 & 8.50 & $1.37 \mathrm{e}-01$ \\
& QSPGS & 178 & 5.56 & $1.37 \mathrm{e}-01$ \\
\hline \multirow{2}{5}{12} & FSPGS & 8911 & 184.75 & $1.37 \mathrm{e}-01$ \\
& HSPGS & 2420 & 30.08 & $1.37 \mathrm{e}-01$ \\
& QSPGS & 655 & 8.36 & $1.37 \mathrm{e}-01$ \\
\hline 1024 & FSPGS & 32602 & 420.11 & $1.37 \mathrm{e}-01$ \\
& HSPGS & 8911 & 189.50 & $1.37 \mathrm{e}-01$ \\
& QSPGS & 2420 & 29.49 & $1.37 \mathrm{e}-01$ \\
\hline 2048 & FSPGS & 116801 & 951.53 & $1.37 \mathrm{e}-01$ \\
& HSPGS & 33318 & 511.32 & $1.37 \mathrm{e}-01$ \\
& QSPGS & 8911 & 188.66 & $1.37 \mathrm{e}-01$ \\
\hline
\end{tabular}




\section{References}

[1] A.R. Abdullah, The Four Point Explicit Decoupled Group (EDG) method: a fast Poisson solver, Int. J. Comput. Math., 38, No 1-2 (1991), 61-70.

[2] O.P. Agrawal, Solution for a fractional diffusion-wave equation defined in a bounded domain, Nonlinear Dynam., 29 (2002), 145-155.

[3] A. Chaves, Fractional diffusion equation to describe Levy flight, Phys. Lett. A, 239, No 1-2 (1998), 13-16.

[4] G. Cheng, T. Huang, X. Cheng, Preconditioned Gauss-Seidel type iterative method for solving linear systems, Appl. Math. Mech., 27, No 9 (2006), $1275-1279$.

[5] J.V.L. Chew, J. Sulaiman, On quarter-sweep finite difference scheme for one-dimensional porous medium equations, Int. J. Appl. Math., 33, No 3 (2020), 439-450.

[6] A. Demir, S. Erman, B. Özgür, E. Korkmaz, Analysis of fractional partial differential equations by Taylor series expansion, Bound. Value Probl., 2013, No 1 (2013), 68-80.

[7] K. Diethelm, A.D. Freed, On the solution of nonlinear fractional order differential equation used in the modeling of viscoelasticity, In: Scientific Computing in Chemical engineering II Computational Fluid Dynamic, Reaction Engineering and Molecular Properties, Springer-Verlag, Heidelberg (1999), 217-224.

[8] D.J. Evans, Group explicit iterative methods for solving large linear systems, Int. J. Comput. Math., 17, No 1 (1985), 81-108.

[9] A.D. Gunawardena, S.K. Jain, L. Snyder, Modified iterative methods for consistent linear systems, Linear Algebra Appl., 154-156 (1991), 123-143.

[10] W. Hackbusch, Iterative Solution of Large Sparse Systems of Equations, Springer-Verlag, New York (1995).

[11] A. Ibrahim, A.R. Abdullah, Solving the two dimensional diffusion equation by the Four Point Explicit Decoupled Group (EDG) iterative method, Int. J. Comput. Math., 58, No 3-4 (1995), 253-256.

[12] T. Kohno, Improving the modified Gauss-Seidel method for Z-matrices, Linear Algebra Appl., 267, No 1-3 (1997), 113-123. 
[13] T.A.M. Langlands, B.I. Henry, The accuracy and stability of an implicit solution method for the fractional diffusion equation, J. Comput. Phys., 205, No 2 (2005), 719-736.

[14] F. Mainardi, Fractals and Fractional Calculus Continuum Mechanics, Springer-Verlag, Heidelberg (1997).

[15] M.M. Meerschaert, C. Tadjeran, Finite difference approximation for fractional advection-dispersion flow equations, J. Comput. Appl. Math., 172, No 1 (2004), 145-155.

[16] M. Othman, A.R. Abdullah, An efficient Four Points Modified Explicit Group Poisson solver, Int. J. Comput. Math., 76, No 2 (2000), 203-217.

[17] I. Podlubny, Fractional Differential Equations, Academic Press, 1999.

[18] R.D. Richtmyer, K.W. Morton, Difference Methods for Initial-Value Problems, Interscience Publishers, New York (1967).

[19] Y. Saad, Iterative Method for Sparse Linear Systems, International Thomas Publishing, Boston (1996).

[20] D.M. Young, Iterative Solution of Large Linear Systems, Academic Press, London (1971).

[21] W.S. Yousif, D.J. Evans, Explicit Group iterative methods for solving elliptic partial differential equations in 3-space dimensions, Int. J. Comput. Math., 18, No 3-4 (1986), 323-340.

[22] S.B. Yuste, L. Acedo, An explicit finite difference method and a new von Neumann-type stability analysis for fractional diffusion equations, SIAM J. Numer. Anal., 42, No 5 (2005), 1862-1874.

[23] S.B. Yuste, Weighted average finite difference method for fractional diffusion equations, J. Comp. Phys., 216 (2006), 264-274.

[24] Y. Zhang, A finite difference method for fractional partial differential equation, Appl. Math. Comput., 215 (2009), 524-529. 
